\title{
Phase conjugation involving incoherent counterpropagating beams in photorefractive media
}

\author{
Mordechai Segev and Amnon Yariv \\ California Institute of Technology, Pasadena, California 91125
}

Received September 3, 1991

\begin{abstract}
We propose and analyze a new type of phase conjugation between counterpropagating beams in photorefractive materials based on diffraction from transmission gratings only. This effect does not require temporal coherence between the counterpropagating beams and may occur spontaneously, with no external seeding, even with wide (frequency) band light sources.
\end{abstract}

Backscattering in photorefractive (PR) materials, as a special case of two-beam coupling, was discovered by Chang and Hellwarth ${ }^{1}$ and has been well studied since then. ${ }^{2}$ This phenomenon involves interaction between two counterpropagating beams, which induce a reflection grating in the volume of the nonlinear medium that simultaneously diffracts. a backward-traveling phase-conjugate wave. Since the effect is a result of interference between the beams, it requires mutual temporal coherence. Moreover, when one beam is self-generated, the input beam is required to have a large coherence length. In principle, the PR backscattering is similar to backscattering in stimulated Brillouin scattering. ${ }^{3}$

We suggest a special phase-conjugate solution involving counterpropagating beams, which may play a key role in the formation of a new type of PR backscattering, based on diffraction from transmission gratings only. In this case, there is no direct interaction between the two counterpropagating beams (no $\lambda / 2$-period, backward-reflecting grating exists), and hence the strict requirement for temporal coherence is removed.

We formulate the PR interaction between the two counterpropagating light beams that are incoherent to each other but are of almost the same frequency. Each beam is assumed to consist of a broad spatial spectrum of plane waves, which interact among themselves and induce large-period transmission gratings in the volume of the nonlinear medium. This type of multiple two-wave mixing interaction is responsible for a variety of self-action effects in PR materials, such as the fanning ${ }^{4}$ and the selffocusing effects in the bulk and funneling effects in waveguides. ${ }^{5}$ We show that while each beam induces transmission gratings and is self-diffracted from them independently of the other beam, it also diffracts from the gratings induced by the spatial components of its counterpropagating mate. In general, the second diffraction process is not phase matched, and hence its efficiency is negligible. An exception is the special case in which the two beams are the phase conjugate of each other, and the principal direction of propagation in the crystalline medium is properly chosen. The self-induced grat- ings of the two beams in this case coincide everywhere in the volume of the nonlinear material, the coherent scattering is reinforced, and the so-called mutual diffraction becomes phase matched. The problem of arranging for a source of backwardtraveling light to be funneled into the phaseconjugate direction may be approached in several different ways. The source may simply be backscattering from scattering centers, in which case the reflectivity would be limited by the total scattering cross section of the passive scatterers, and the signal-to-noise ratio would be low close to the phaseconjugate mirror but increase as the distance squared farther away. A second method would be to use strong fanning, ${ }^{4}$ starting from forward antifunneling and ending in backward funneling (Fig. 1). A third method would be to use external seeding, in which case the device would be a degenerate version of the double phase-conjugate mirror. ${ }^{6}$ In discussing this third possibility we note its contribution to the understanding of the lack of cross talk ${ }^{6}$ in this device.

Since the key to this scattering process is grating formation by the continuum of Fourier (plane-wave) components of each beam, we cannot resort to the two-plane-wave analysis commonly applied to PR materials. We present a new general formalism that includes the necessary transverse beam spatial structure and permits a complete treatment of image-bearing beam coupling in PR materials. We start our formalism with the nonlinear wave equation applied to the case of two quasi-monochromatic counterpropagating optical beams, of the same wavelength $(\lambda)$ and polarization, which travel in the positive and the negative directions of an arbitrary axis $z$. For simplicity, we consider a twodimensional $(y-z)$ case (we note that our analysis remains valid when this additional dimension is included). The total light electric field in that case is

$$
\begin{aligned}
E(y, z, t)= & A_{1}(y, z) \exp [i(k z-\omega t)] \\
& +A_{2}(y, z) \exp [-i(k z+\omega t)]+\text { c.c. }
\end{aligned}
$$

Assuming a temporal steady state and a small (lightinduced) perturbation in the refractive index $\delta n(y, z)$ due to nonlinear wave interaction, we obtain the non- 


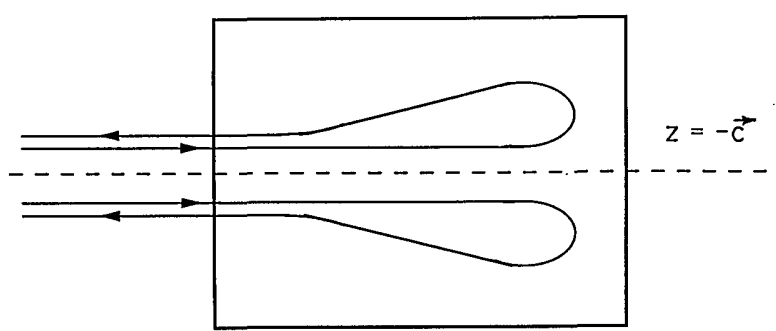

Fig. 1. Self-generated phase-conjugate backscattering, seeded by fanning: the forward antifunneling is converted into backward funneling.

linear paraxial wave equation for $A_{1}$ and $A_{2}$,

$$
\begin{array}{r}
\left(\frac{\partial^{2} A_{1}}{\partial y^{2}}+2 i k \frac{\partial A_{1}}{\partial z}\right) e^{i k z}+\left(\frac{\partial^{2} A_{2}}{\partial y^{2}}-2 i k \frac{\partial A_{2}}{\partial z}\right) e^{-i k z} \\
=-\frac{2 k^{2}}{n_{0}} \delta n(y, z)\left(A_{1} e^{i k z}+A_{2} e^{-i k z}\right),
\end{array}
$$

where $n_{0}$ is the unperturbed index of refraction.

In this analysis, we are interested in transmission gratings only. The reflection gratings can be eliminated by taking $A_{1}$ and $A_{2}$ to be temporally mutually incoherent. In this case the only light-induced gratings in the material volume are those formed by interference between spatial components of each beam, independently of the presence of the other one. This causes the induced gratings $\delta n(y, z)$ to vary slowly with $z$, and we can reduce Eq. (2) into

$$
\begin{gathered}
\frac{\partial^{2} A_{1}}{\partial y^{2}}+2 i k \frac{\partial A_{1}}{\partial z}=-\frac{2 k^{2}}{n_{0}} \delta n(y, z) A_{1}, \\
\frac{\partial^{2} A_{2}{ }^{*}}{\partial y^{2}}+2 i k \frac{\partial A_{2}{ }^{*}}{\partial z}=-\frac{2 k^{2}}{n_{0}} \delta n(y, z) A_{2}{ }^{*} .
\end{gathered}
$$

In our case, where the cross grating $A_{1} A_{2}{ }^{*}$ terms average out to zero, the only mutual interaction between the beams may be the scattering of each beam from the gratings induced by its counterpropagating mate.

We define $f_{i}(q, z)$ as the spatial frequency (angular) distribution of the complex amplitude $A_{i}(y, z)$ (of beam $i$, where $i=1,2$ ) by the relation

$$
f_{i}(q, z)=\int_{-\infty}^{\infty} A_{i}(y, z) e^{-i q y} e^{-i\left(k_{z}-k\right) z} \mathrm{~d} y,
$$

where $q$ and $k_{z}$ are the projections (on the $y$ and $z$ axes, respectively) of the wave vector $\mathbf{k}$ that characterizes the spatial component (plane wave) that propagates at an angle $\alpha$ with respect to the optical axis $z$. The spatial frequency $q$ is related to $\alpha$ by $q=2 \pi n \sin (\alpha) / \lambda$ and $k_{z}$ satisfies $k_{z}=+\sqrt{k^{2}-q^{2}}$.

The nonlinear term $\delta n(y, z)$ is obtained from a consideration of the mixing process between two plane waves. When only one pair of plane waves of field amplitudes $a_{1}(z)$ and $a_{2}(z)$ (and spatial frequencies $q_{1}$ and $q_{2}$ ) are present, they induce an index grating $\delta n(y, z)$ that is proportional to the time-averaged interference pattern between them. The proportionality coefficient is a complex factor $\widehat{\delta n}\left(q_{1}, q_{2}\right)$, which represents the PR coupling coefficient between two waves propagating at $\alpha_{1}$ and $\alpha_{2}$, given the material properties, the orientation of the crystalline medium, and the polarization of the waves. In this simple case, $\delta n(y, z)$ is

$$
\begin{aligned}
& \delta n(y, z)=\frac{1}{I_{0}}\left(a_{1}(z) \exp \left\{i\left[q_{1} y+\left(k_{z 1}-k\right) z\right]\right\} a_{2}{ }^{*}(z)\right. \\
& \left.\times \exp \left\{-i\left[q_{2} y+\left(k_{z 2}-k\right) z\right]\right\} \widehat{\delta n}\left(q_{1}, q_{2}\right)+\text { c.c. }\right),
\end{aligned}
$$

where $I_{0}=\left|a_{1}\right|^{2}+\left|a_{2}\right|^{2}$ (the absolute light intensity). Since $\delta n(y, z)$ is real, $\widehat{\delta n}\left(q_{1}, q_{2}\right)=\widehat{\delta n} *\left(q_{2}, q_{1}\right)$. The coupling coefficient is a product of two terms, $r_{\text {eff }}\left(q_{1}, q_{2}\right)$, which is a scalar product of the material's electro-optic tensor, and $E_{m}\left(q_{1}, q_{2}\right)$, which is the coefficient of the induced space-charge field. ${ }^{5}$

When more than two plane waves are present, the term $\delta n(y, z)$ involves a summation over all the interacting plane waves. For a given light beam $A(y, z)$, which consists of a continuous spatial spectrum of plane waves $f(q, z)$, this summation takes an integral form,

$$
\begin{array}{r}
\delta n(y, z)=\frac{1}{F_{0}} \int \mathrm{d} q_{1} \int \mathrm{d} q_{2} f\left(q_{1}, z\right) f^{*}\left(q_{2}, z\right) \exp \left(i \left\{\left(q_{1}\right.\right.\right. \\
\left.\left.\left.-q_{2}\right) y+\left[\left(k_{z 1}-k\right)-\left(k_{z 2}-k\right)\right] z\right\}\right) \widehat{\delta n}\left(q_{1}, q_{2}\right),
\end{array}
$$

where $F_{0}=\int_{-\infty}^{\infty}|f(q, z)|^{2} \mathrm{~d} q$ is the total light power ${ }^{5}$ (averaged over $y$ ) and $k_{z i}=+\sqrt{k^{2}-q_{i}^{2}}$.

We formulate here the interaction between two counterpropagating beams, as described by Eq. (1). In our case, the term $f\left(q_{i}, z\right) \exp \left\{i\left[q_{i} y+\left(k_{z i}-k\right) z\right]\right\}$ in Eq. (6) is replaced by $f_{1}\left(q_{i}, z\right) \exp \left\{i\left[q_{i} y+\left(k_{z i}-\right.\right.\right.$ $k) z]\}+f_{2}^{*}\left(q_{i}, z\right) \exp \left\{-i\left[q_{i} y+\left(k_{z i}-k\right) z\right]\right\}$. Substitution of this expression into Eq. (6) and the resulting $\delta n(y, z)$ term into Eqs. (3) gives a coupled set of equations for the field amplitudes $A_{1}$ and $A_{2}$ and their Fourier transforms $f_{1}$ and $f_{2}$. We simplify Eqs. (3) by Fourier transforming them: multiplying the first relation of Eqs. (3) by $\exp \left\{-i\left[q y+\left(k_{z}-k\right) z\right]\right\}$ and the second relation by $\exp \left\{i\left[q y+\left(k_{z}-k\right) z\right]\right\}$ and integrating over the whole transverse space (the $y$ coordinate) with Eq. (4) and the above relations. The left-hand sides of Eqs. (3) are transformed in a trivial way, but the right-hand sides need further consideration. The Fourier transform of the righthand side of the first relation of Eqs. (3) is

$$
\begin{aligned}
& -\frac{2 k^{2}}{n_{0} F_{0}} \int_{-\infty}^{\infty} \mathrm{d} y\left\{\int f_{1}\left(q_{3}, z\right) \exp \left[i\left(q_{3} y+k_{z 3} z\right)\right] \mathrm{d} q_{3}\right\} \\
& \times \exp \left[-i\left(q y+k_{z} z\right)\right] \int \mathrm{d} q_{1} \int \mathrm{d} q_{2} \widehat{\delta n}\left(q_{1}, q_{2}\right) \\
& \times\left\{f_{1}\left(q_{1}, z\right) f_{1}^{*}\left(q_{2}, z\right) \exp \left[i\left(q_{1}-q_{2}\right) y\right]\right. \\
& \times \exp \left[i\left(k_{z 1}-k_{z 2}\right) z\right]+f_{2}^{*}\left(q_{1}, z\right) f_{2}\left(q_{2}, z\right) \\
& \left.\times \exp \left[-i\left(q_{1}-q_{2}\right) y\right] \exp \left[-i\left(k_{z 1}-k_{z 2}\right) z\right]\right\} .
\end{aligned}
$$

Integration over $y$ first gives rise to two momentum conservation laws. The first one is conservation of the momentum perpendicular to the optical axis $(z)$, which is represented here by a delta function, unique to each of the two terms in expression (7). The second law is the conservation of the momentum parallel to $z$, which is known as the phase-matching condition in thick (volume) holograms and is represented by the collection of all the $\exp \left(i k_{z i} z\right)$ terms.

The first term in expression (7) contributes only to self-scattering processes, since only $f_{1}$ is involved. The resulting delta function from the integra- 
tion over $y$ is $\delta\left(q-q_{1}+q_{2}-q_{3}\right)$, and the phasematching term is $\exp \left[-i\left(k_{z}-k_{z 1}+k_{z 2}-k_{z 3}\right) z\right]$. The delta function is satisfied automatically when $q_{1}=q$ and $q_{3}=q_{2}$ and the exponential term becomes unity. This result is obtained by integrating the whole equation over a small interval $\mathrm{d} z$, which is larger than a wavelength, and considering only the main contribution [taking advantage of $f(q, z)$ being a slowly varying function of $z$ ]. The result is the first term on the left-hand side of Eq. (8), where $F_{1}\left(q^{\prime}, z\right)=\left|f_{1}\left(q^{\prime}, z\right)\right|^{2}$. This self-interaction nonlinear term is responsible for the PR self-action phenomen ${ }^{5,6}$ and is always phase matched, since it involves scattering of a beam from its own grating.

The second term in expression (7) represents mutual scattering, where one beam is diffracted from gratings induced solely by its mate. Two waves $\left(q_{1}\right.$ and $q_{2}$ ) form an induced grating, and a third wave $q_{3}$, antiparallel to $q_{2}$, is diffracted from it in a direction antiparallel to $q$. Under these conditions, $q_{1}=-q$ and $q_{3}=-q_{2}$, the perpendicular momentum conservation law [represented by $\delta\left(q+q_{1}-q_{2}-q_{3}\right)$ ] is obeyed, but phase matching is not guaranteed. The resultant mutual scattering is represented by the last term on the left-hand side of Eq. (8). The complete nonlinear equation for $f_{1}(q, z)$ is

$$
\begin{aligned}
& {\left[q^{2}+2 k\left(k_{z}-k\right)\right] f_{1}(q, z)-2 i k \frac{\partial f_{1}(q, z)}{\partial z}} \\
& =\frac{2 k^{2}}{n_{0} F_{0}}\left[f_{1}(q, z) \int F_{1}\left(q^{\prime}, z\right) \widehat{\delta n}\left(q, q^{\prime}\right) \mathrm{d} q^{\prime}\right. \\
& \quad+f_{2}^{*}(-q, z) \exp \left(-2 i k_{z} z\right) \int f_{1}\left(-q^{\prime}, z\right) f_{2}\left(q^{\prime}, z\right) \\
& \left.\times \exp \left(2 i k_{z}^{\prime} z\right) \widehat{\delta n}\left(-q, q^{\prime}\right) \mathrm{d} q^{\prime}\right] .
\end{aligned}
$$

A similar procedure, when applied to the second relation of Eqs. (3), yields an analogous equation. In general, the first term on the right-hand side of Eq. (8) changes slowly with $z$, but the second terms (which correspond to the mutual scattering) contain an exponential function that changes rapidly and average out to zero for an interaction length larger than the wavelength.

In one special case, when the phase-matching requirements are fulfilled, we get significant mutual scattering, and each beam is efficiently scattered from the gratings induced by its mate. We show that if the two beams $A_{1}(y, z)$ and $A_{2}(y, z)$ are the phase conjugates of each other, this mutual scattering is phase matched everywhere. By substituting $A_{1}(y, z)=A_{2}{ }^{*}(y, z)$ for all $y$ and $z$ into Eq. (4), we obtain an equivalent requirement, for all $q, z$,

$$
f_{1}(q, z)=f_{2}^{*}(-q, z) \exp \left[-2 i\left(k_{z}-k\right) z\right] \text {. }
$$

Substitution of this relation in Eqs. (8) ensures immediate phase matching for the mutual scattering terms in both equations,

$$
\begin{array}{r}
{\left[q^{2}+2 k\left(k_{z}-k\right)\right] f_{1}(q, z)-2 i k \frac{\partial f_{1}(q, z)}{\partial z}=\frac{2 k^{2}}{n_{0} F_{0}} f_{1}(q, z)} \\
\times \int F_{1}\left(q^{\prime}, z\right)\left[\widehat{\delta n}\left(q, q^{\prime}\right)+\widehat{\delta n}\left(-q,-q^{\prime}\right)\right] \mathrm{d} q^{\prime} \quad(10)
\end{array}
$$

and an analogous equation for $f_{2}{ }^{*}(q, z)$. It follows straightforwardly that if $f_{1}(q, z)$ satisfies Eq. (10) and obeys relation (9), then the equation for $f_{2}{ }^{*}$ is automatically satisfied. Up to this point we have proven that a PR medium supports phase conjugation between two mutually incoherent beams by relaying on transmission gratings only. Without the presence of a seeding mechanism, which may play the role of an intermediate, the significance of this effect is limited. Amplified noise (fanning ${ }^{4}$ ) may play an important role of seeding the $A_{2}$ beam, by optimization of the fanning stimulated scattering process, into the preferential directions dictated by $A_{1}$. When the gratings induced by both beams are identical, i.e., $\widehat{\delta n}\left(q, q^{\prime}\right)=\widehat{\delta n}\left(-q,-q^{\prime}\right)$ for all $q$ and $q^{\prime}$, the material not only supports the phaseconjugated beams, but also prefers them over other possibilities by stimulating higher diffraction efficiency from their gratings than from any other set that may be present in the medium. In this case we predict a resultant self-seeded backscattering process [note that it requires $\widehat{\delta n}\left(q, q^{\prime}\right)$ to have an imaginary part, to permit the fanning evolution]. The last condition is satisfied for an imaginary $\hat{\delta}\left(q, q^{\prime}\right)$ when the $z$ axis coincides with the $\mathbf{c}$ axis in several uniaxial crystals. ${ }^{5}$ This stimulated scattering has the potential of becoming dominant in the wave mixing process and may support self-generation of one of the beams (see Fig. 1). Moreover, when externally seeded, it may be considered as a degenerate case of a double phase-conjugate mirror, and it suggests an explanation for the lack of cross talk between the interacting pump beams in this device. ${ }^{6}$

In conclusion, we have formulated wave mixing in PR media in a way that accounts for the nonlinear interaction among the Fourier (plane-wave) components of a given beam. We predict the existence of a new backscattering phenomenon, which occurs even between mutually incoherent beams and may be self-generated. We note that our formalism is essential for accurate calculations and a correct understanding of any beam-coupling effects whenever the beams bear any pictorial information other than being pure plane waves.

\section{References}

1. T. Y. Chang and R. W. Hellwarth, Opt. Lett. 10, 408 (1985).

2. See, for example, G. C. Valley, in Digest of Conference on Lasers and Electro-Optics (Optical Society of America, Washington, D.C., 1990), paper CPDP 22, and references therein.

3. B. Y. Zel'dovich, N. F. Pilipetsky, and V. V. Shkunov, Principles of Phase Conjugation (Springer-Verlag, New York, 1989).

4. M. Segev, Y. Ophir, and B. Fischer, Opt. Commun. 77, 265 (1990).

5. M. Segev, Y. Ophir, and B. Fischer, Appl. Phys. Lett. 56, 1086 (1990); B. Fischer and M. Segev, Appl. Phys. Lett. 54, 684 (1989).

6. S. Weiss, S. Sternklar, and B. Fischer, Opt. Lett. 12, 114 (1987), 